Cipango $\begin{aligned} & \text { Cipango } \\ & \text { Cahiers d'études japonaises }\end{aligned}$

19 | 2012

Le Japon et le fait colonial II

\title{
Katsumata Shizuo, Ikki, coalitions, ligues et révoltes dans le Japon d'autrefois
}

\section{Guillaume Carré}

\section{(2) OpenEdition}

1 Journals

\section{Édition électronique}

URL : https://journals.openedition.org/cipango/1723

DOI : $10.4000 /$ cipango. 1723

ISSN : 2260-7706

Éditeur

INALCO

\section{Édition imprimée}

Date de publication : 30 octobre 2012

ISBN : 978-2-85831-204-7

ISSN : $1164-5857$

\section{Référence électronique}

Guillaume Carré, «Katsumata Shizuo, Ikki, coalitions, liques et révoltes dans le Japon d'autrefois », Cipango [En ligne], 19 | 2012, mis en ligne le 06 mai 2014, consulté le 30 juin 2021. URL : http:// journals.openedition.org/cipango/1723 ; DOI : https://doi.org/10.4000/cipango.1723

Ce document a été généré automatiquement le 30 juin 2021.

\section{(c) (7) (9)}

Cipango est mis à disposition selon les termes de la Licence Creative Commons Attribution - Pas d'Utilisation Commerciale 4.0 International. 


\title{
Katsumata Shizuo, Ikki, coalitions, ligues et révoltes dans le Japon d'autrefois
}

\author{
Guillaume Carré
}

\section{RÉFÉRENCE}

Katsumata Shizuo, Ikki, coalitions, ligues et révoltes dans le Japon d'autrefois, introduction, traduction et notes de Pierre-François Souyri, Paris, CNRS éditions, 2011, 268 p. ISBN : 978-2-271-07274-0.

1 Il arrive que des historiens japonais, en particulier des médiévistes, affichent leur positionnement par rapport à une « histoire sociale » (shakaishi), et affirment parfois ne pas en relever, alors même que l'objet de leurs travaux n'est rien d'autre que le passé de la société japonaise. Nous sommes une fois de plus pris au piège des mots et de leurs traductions ; car si «shakaishi» est bien l'équivalent lexical d'" histoire sociale », cette expression n'a pas forcément la même résonnance dans les contextes académiques français et japonais. Au Japon en effet, l'« histoire sociale » est souvent entendue, dans une relation plus ou moins antagoniste, par rapport à une " histoire des mouvements sociaux", ou encore une "histoire économique et sociale", voire une "histoire d'après-guerre ", ces expressions désignant en réalité les courants principaux de l'historiographie marxiste qui domina le monde académique japonais jusque dans les années 1970. La mouvance intellectuelle connue sous le nom d'« histoire sociale» regroupe donc des universitaires, qui sous des formes directes ou non, contestèrent l'hégémonie des méthodes du matérialisme historique, pour trouver une audience de plus en plus large à partir des années 1980 .

2 Naturellement, on n'a pas attendu la fin du $\mathrm{xx}^{\mathrm{e}}$ siècle pour écrire de l'histoire sociale au Japon, puisqu'à vrai dire, les historiens marxistes ne faisaient pas autre chose. Mais ceux qui se retrouvèrent classés dans la mouvance de «l'histoire sociale » japonaise 
recherchaient de nouvelles approches des réalités sociales passées, et souhaitaient imposer dans le champ académique de nouveaux objets d'études. Deux influences majeures alimentèrent ce courant historique : tout d'abord, les études folkloriques (minzokugaku), qui avaient connu un développement important au Japon depuis les années 1920. En s'attachant non seulement à l'étude des traditions japonaises, mais aussi à celle des représentations du monde qui les sous-tendaient, les études folkloriques offraient de nouvelles approches du "peuple» pour des historiens qui souhaitaient prendre leurs distances avec l'orthodoxie marxiste. Le représentant le plus connu de cette tendance est le médiéviste Amino Yoshihiko, qui, après sa rupture dans les années 1950 avec le courant dominant de sa discipline, celui d'Ishimoda Shō et de ses épigones, s'inspira de plus en plus des travaux de folkloristes comme Yanagita Kunio ou Miyamoto Tsuneichi, pour remettre en cause des conceptions liant société japonaise et civilisation rizicole.

3 Mais les tenants de l'« histoire sociale » japonaise, qu'ils l'aient reconnu explicitement ou non, furent aussi influencés par les évolutions de leur discipline en Occident. On le perçoit avec l'introduction de nouvelles méthodes, comme l'archéologie médiévale, ou de nouveaux objets, avec par exemple le développement de l'analyse iconographique ; mais c'est aussi à cette époque, vers les années 1980, que certaines des problématiques de la «nouvelle histoire » portée par l'école des Annales, furent introduites au Japon par des historiens occidentalistes comme Abe Kin'ya (histoire médiévale allemande), ou le spécialiste de l'Ancien Régime français Ninomiya Hiroyuki. Contre une lecture de la société régie par les seuls rapports de classes et de production, ces historiens légitimèrent une recherche plus respectueuse de la variété des contextes et des groupes sociaux, des pratiques, des formes de sociabilité et des mentalités.

$4 \mathrm{Au}$ croisement de ces tendances, on trouve aussi des historiens moins marqués par le catéchisme marxiste qu'une bonne part de leurs collègues, car moins préoccupés par la catégorisation des classes sociales que par les conceptions du droit, de la loi, et de la justice dans le Japon médiéval, et par leur formalisation, comme Kasamatsu Hiroshi, ou Katsumata Shizuo, l'auteur de l'ouvrage intitulé Ikki, dont la traduction en français fait l'objet de la présente recension. On agrège donc dans cette "histoire sociale", expression devenue en japonais un équivalent d'une anthropologie historique locale, des chercheurs aux références et horizons en fin de compte assez divers. Mais ces nouvelles propositions vinrent à point nommé pour nourrir la réflexion académique alors que l'essoufflement des schémas du matérialisme historique devenait de plus en plus évident, et qu'un besoin de renouvellement se faisait sentir même chez des historiens de formation marxisante.

5 L'ouvrage intitulé $I k k i$, publié en 1982, est donc paru à un moment de basculement des sciences historiques japonaises, et son retentissement montre qu'il a pu être lu comme une sorte de manifeste de cette nouvelle « histoire sociale ». Ce texte ne prend pourtant pas l'apparence d'un ouvrage de recherche académique : il a été conçu pour une édition de poche (shinsho), volontairement accessible, sinon grand public. Quant à l'objet d'étude, il n'avait à première vue rien de bien nouveau: dans son introduction, le traducteur de Katsumata, Pierre-François Souyri, rappelle que l'intérêt pour les mouvements de protestations populaires (ikki) dans les sciences sociales japonaises remonte aux " émeutes du riz » qui secouèrent l'archipel en 1918. Ces révoltes étaient naturellement un sujet qu'affectionnaient les historiens marxistes, et d'ailleurs Katsumata a fait un usage abondant des travaux de ses prédécesseurs : ceux-ci avaient 
déjà recensé les « émotions " populaires sur la longue durée, et on trouve dans Ikki de nombreuses références au choix de textes dirigé par Shōji Kichinosuke, Hayashi Hajime et Yasumaru Yoshio, intitulé Pensée des mouvements populaires (Minshū undō no shisō, collection Nihon shisō Taikei, publié en 1970). Les soulèvements paysans ou urbains ne pouvaient en effet que focaliser l'attention d'historiens à la recherche des indices d'une dynamique de changement social mue par la lutte des classes : ainsi, les autonomies populaires de la fin du Moyen Âge avaient été interprétées en 1953 par Araki Moriaki, comme la manifestation de l'émergence et de l'émancipation d'une nouvelle catégorie sociale, celle des guerriers ruraux, qui auraient imposé leur domination au terme d'un processus révolutionnaire achevé par l'établissement du cadastre de Toyotomi Hideyoshi.

6 La révolution portée par l'entreprise de Katsumata Shizuo était cependant d'un autre ordre. Son intérêt pour les ikki ne portait pas en priorité sur les systèmes socioéconomiques et les rapports de domination au milieu desquels fermentaient les révoltes populaires. L'objet de sa réflexion est bien l'ikki en tant que telle, c'est-à-dire la «ligue jurée", sens originel d'une expression qui a fini par devenir synonyme de "révolte ", et même de "révolte paysanne ", mais qui a été employée sur une longue période allant des débuts du Moyen Âge à la fin de la période d'Edo, pour désigner des modes de coalition, de revendication et de résistance utilisés par des communautés qui estimaient leurs droits violés ou menacés. La première audace de Katsumata était donc de considérer l'ikki comme un phénomène global qu'il convenait d'étudier en dépassant la coupure disciplinaire entre histoire médiévale et histoire de la période d'Edo, pour faire ressortir les continuités dans des formes et des mobiles de protestation qui connurent naturellement aussi de profondes évolutions entre les XIII et XIX ${ }^{e}$ siècles.

7 Cette continuité, Katsumata la décèle non seulement dans la persistance du terme ikki jusqu'à l'orée de l'ère industrielle, mais aussi dans l'héritage des pratiques qui régissaient ce type de coalition : prestation de serment devant les divinités, ingestion d'une « eau sacrée " par les conjurés, port de déguisements divers... L'attention portée aux comportements et aux éléments de mise en scène est une des grandes originalités de Katsumata, mais sa finalité n'est pas uniquement descriptive: l'examen de la transmission des formes permet en effet de reconstruire une généalogie des modes de protestation, et conduit à une réflexion sur les conceptions de l'ordre social, du droit individuel et collectif, du rapport au sacré qui légitimaient le défi envers l'autorité, et assuraient la cohésion des mouvements de désobéissance.

8 Sous le couvert d'un déroulement chronologique classique, partant de l'époque Kamakura pour finir à l'ère Meiji, c'est donc une histoire des mentalités sur la longue durée que s'est proposé d'écrire Katsumata Shizuo. Et de plus, loin de considérer l'ikki seulement comme la manifestation d'une révolte contre des formes d'oppression, il entend montrer que ces formes de coalition s'enracinent dans des conceptions de la prise de décision et de la justice répandues dans toutes les classes et conditions sociales. Dans le droit fil de ses travaux sur le droit médiéval, qui l'amènent naturellement à recourir à plusieurs reprises à des sources juridiques ou judiciaires, Katsumata commence par s'interroger sur les modes de délibération dans le Conseil d'État du shogounat de Kamakura, au XII siècle. Les pratiques de coalition caractéristiques de l'ikki tiennent en effet leurs origines, selon l'auteur, des formes qui régulaient les prises de décisions et l'action collective dans des groupes comme les assemblées monastiques ou les associations entre guerriers au début du Moyen Âge : la 
délibération entre les membres de la communauté devait en principe conduire à l'adoption d'une attitude reconnue comme juste par la majorité, et qui devait être considérée dès lors comme reflétant un sentiment unanimement partagé (ichimidōshin).

La décision s'imposait donc à tous les membres du groupe, et la contrainte était assurée non seulement par la pression collective, mais aussi par le serment devant les divinités qui assurait en même temps la légitimité de la position ainsi arrêtée. En engageant la responsabilité individuelle et solidaire devant les puissances surnaturelles, la ligue ainsi formée manifestait le caractère indiscutable et fondé de la décision ou de la revendication (d'où la fiction de la position «unanimiste»), même quand elles s'opposaient frontalement à une autorité sociale, politique ou religieuse. L'implication des divinités dans les querelles humaines était d'ailleurs en soi un moyen de pression à des époques où l'efficacité redoutable du recours au surnaturel ne faisait de doute pour personne, et les moines de l'époque de Heian ne se privaient pas d'en abuser, comme le rappelle Katsumata.

Aux sources de l'ikki, on trouve donc ces alliances entre individus liés par des intérêts et un territoire communs à défendre, et qui structuraient par exemple certaines compagnies militaires féodales du Moyen Âge, dont Katsumata décrit le fonctionnement. Et comme jusqu'à la fin du $\mathrm{XVI}^{\mathrm{e}}$ siècle, la masse des guerriers résidait dans les campagnes, les ligues finirent par constituer un mode d'organisation courant des populations rurales. Bien que la constitution de telles ligues ait pu déboucher sur de véritables rébellions de grande ampleur, en particulier au xvI siècle, l'ikki, loin d'être une explosion de colère incontrôlée, prenait souvent la forme de mouvements bien encadrés par toute une organisation communautaire, avec ses règles, ses rituels, des objectifs précis, des cibles privilégiées désignées à la vindicte populaire; et les références à des pratiques plus ou moins magiques, religieuses ou coutumières, pouvaient fournir tout un arsenal de symboles ou d'actions codifiées permettant d'éviter un déchaînement de violence. Katsumata détaille ainsi plusieurs formes de résistance des villageois face à l'autorité seigneuriale, qu'il s'agisse de l'étalage de bambous sur le sol pour interdire un accès, ou de la désertion collective d'une localité. Les ligueurs pouvaient donc jouer sur une gamme allant de la manifestation bruyante à l'émeute, et tenter ainsi de faire prévaloir leur point de vue en graduant leur action et leurs revendications en fonction du rapport de force avec le pouvoir, et de la signification qu'ils entendaient donner au mouvement.

11 On peut dresser une certaine typologie des insurrections populaires en fonction des périodes historiques et des régions: mouvements pour l'abolition des dettes de l'époque Muromachi ( $\mathrm{xv}^{\mathrm{e}}$ siècle), rejet de la domination des grands féodaux structuré autour de sectes bouddhistes au XVI ${ }^{\mathrm{e}}$ siècle, agitation anti-fiscale ou contestation des politiques seigneuriales lors de la période d'Edo, paniques millénaristes de la fin du shogounat des Tokugawa... Sans compter les explosions de ressentiment contre les " accapareurs » ou les «monopoleurs» lors des disettes ou des famines, du XvIII et jusqu'à l'ère Meiji. La certitude des acteurs de ces révoltes de défendre leur bon droit se fondait sur un rapport ancestral à la propriété foncière et au patrimoine, et sur la force des rapports communautaires, à la fois solidarité et contrainte pesant de génération et génération. L'attachement au terroir, au patrimoine foncier mis en valeur par les ancêtres et sur lequel se fondait la subsistance et parfois l'identité, que ce soit dans les monastères ou au sein de la "mesnie» des petits seigneurs ruraux, puis de la 
communauté villageoise, est le grand moteur des mécontements, en particulier au Moyen Âge. Il pousse à dénier toute valeur définitive à la transmission hors des règles acceptées par l'ordre communautaire, d'où le refus fréquent de la cession pour dettes et la demande récurrente de leur annulation pouvant mener à des soulèvements violents, comme dans la région de Kyōto au xve siècle.

Cette mentalité d'ailleurs, rappelons-le, ne reflétait pas seulement les frustrations de rustres appauvris : l'aristocratie religieuse ou guerrière, dont l'identité reposait sur la domination territoriale, ne pouvait reconnaître pleinement la possibilité de l'aliénation de la possession foncière et des droits afférents pour payer des créanciers, dans un contexte social où puissance de la monnaie et légitimité de la propriété appartenaient encore à des ordres de valeur différents et concurrents. De là les annulations générales de dettes prononcées par les autorités shogounales du Moyen Âge, en partie pour contenter les petits seigneurs ruraux qui prenaient la tête des ligues; et à l'époque d'Edo, alors que les autorités guerrières vont renoncer à cette politique, sauf épisodiquement en faveur des vassaux ou dans le cadre de banqueroutes de principautés seigneuriales, la mémoire en demeurera fermement implanté chez les masses paysannes, en continuant à les mobiliser dans les périodes de crise contre les usuriers ou tous les grossiums accusés de manquer à la solidarité communautaire.

Tout en remettant subrepticement en cause les a priori méthodologiques du matérialisme historique, il est évident que Katsumata, tout comme Amino, tient fermement son histoire des pratiques et des mentalités dans la poursuite de l'écriture d'une histoire populaire, ou pour le dire autrement une histoire "du peuple», sans dériver vers une surreprésentation de catégories élitaires ou intellectuelles mieux documentées, comme cela a pu être parfois le cas en Europe. La singularisation par rapport aux courants historiographiques du marxisme ne signifie donc pas un renoncement à cette ambition essentielle des historiens marxisants plus ou moins orthodoxes, et qui continua par la suite à animer l'historiographie japonaise; une préoccupation d'ailleurs proche du souci des folkloristes de décrire la vie des gens ordinaires. Pour Katsumata, il s'agit avant tout de ne pas subordonner l'analyse des coalitions populaires à une téléologie ou à un cadre analytique prédéfini par les rapports de production, mais de les resituer dans une perspective élargie et évolutive des représentations soutenant les conceptions du droit, au-delà des clivages de conditions ou de classes.

L'intérêt de Katsumata se porte aussi sur des catégories sociales déshéritées qui paradoxalement préoccupaient peu les historiens marxistes orthodoxes, mais passionnaient Amino Yoshihiko : en soulignant que les apparences et attitudes plus ou moins extravagantes adoptées par les manifestants manifestaient leur volonté d'échapper momentanément à un ordre social qui les maintenait d'habitude sous le poids de ses hiérarchies, l'auteur rapproche ces comportements de ceux des parias, les «non-humains » (hinin) discriminés qui incarnaient la marginalité sous sa forme la plus radicale.

Mais en fait, les rapports entretenus par Katsumata avec l'historiographie marxiste paraissent plus complexes que pourrait le donner à penser son recours aux études folkloriques ou ses citations de l'Essai sur le don de Marcel Mauss. Tout comme Amino, ses perspectives novatrices sur la société médiévale contrastent avec sa compréhension des structures et du fonctionnement du Japon de l'époque d'Edo, qui reste très tributaire de l'historiographie marxisante qui dominait la discipline dans les 
années 1970. L'explication de l'évolution des mouvements de protestation populaire sous les Tokugawa est, par exemple, un décalque des travaux de Sasaki Junnosuke. Si donc Katsumata, lorsqu'il nous parle du Moyen Âge, ne se laisse pas corseter par des catégorisations trop rigides dans son appréhension des réalités sociales et de la production des imaginaires, sa vision de l'époque d'Edo reste pourtant celle de populations strictement encadrées dans des statuts imposés par l'autorité guerrière. Katsumata reconnaît d'ailleurs qu'il a construit ses réflexions sur la période prémoderne en s'appuyant sur un socle bâti par d'autres chercheurs, et cela ne l'empêche nullement de renouveler le regard porté sur les soulèvements populaires de cette époque, du point de vue de l'héritage et de l'évolution des pratiques de manifestation et de mobilisation surtout. Mais ce regard de médiéviste porté sur la période d'Edo n'échappe pas à certaines distorsions, réduisant le Japon des Tokugawa une période de perte de liberté populaire. Cet aspect reste toutefois plus discret, peutêtre plus prudent, chez Katsumata Shizuo que chez Amino Yoshihiko, dont Asao Naohiro faisait l'un des héritiers d'une légende noire de la période d'Edo remontant à l'ère Meiji.

D'autre part, en recourant aux acquis et méthodes des études folkloriques, Katsumata Shizuo s'est bien doté d'un outil lui permettant de renouveler les analyses sur les protestations et émeutes populaires, mais on ne peut se défaire de l'impression qu'il laisse ça et là contaminer son approche par la désinvolture avec laquelle les processus historiques sont parfois traités par une certaine anthropologie. Que des manières de se costumer ou de modifier son apparence, que des formes assez étranges de manifester une opposition au pouvoir, plongent leurs racines dans des temps médiévaux reculés, et qu'ils aient pu survivre jusqu'à l'aube de l'ère Meiji, l'auteur en fait la démonstration avec une précision qui emporte l'adhésion. Mais pour autant que les pratiques perdurent, cela signifie-t-il forcément que la compréhension de leur signification n'est pas altérée? On défile bien en Bretagne pour les pardons de nos jours comme au $\mathrm{XVIII}^{\mathrm{e}}$ siècle, mais certainement pas avec la même perception de l'évènement et de son cérémonial. Et ce qui vaut pour la religion vaut sans doute pour toutes les institutions sociales. D'ailleurs à l'époque où Katsumata écrivait Ikki, les études folkloriques ellesmêmes accordaient une attention nouvelle à l'évolution et au changement de signification et de perception des pratiques et coutumes populaires, dans la lignée des travaux sur les sociétés urbaines de Miyata Noboru. Et puis, la signification première des rites et pratiques, pour autant qu'on puisse la situer, parait parfois plutôt une reconstruction de Katsumata, procédant plus ou moins explicitement par interprétation ou par analogie.

D'une manière générale, l'anthropologie historique japonaise laisse l'impression de masses rurales vivant dans un Moyen Âge saturé de sacré, ou tout est en rapport avec le divin, le surnaturel, ou le tabou. Peut-être en allait-il ainsi. Mais de même que l'on a pu légitimement se demander si les Grecs ont toujours cru à leurs mythes, on peut aussi questionner le rapport que des Japonais médiévaux ont eu au surnaturel : au fond, jurer ses Grands Dieux, jouer les démons de carnaval lorsqu'on manifeste, faire bénir les armes, est-ce le signe que l'on vit continuellement plongé dans une terreur sacrée ? La revendication sur les terres, la protestation contre le fisc et les redevances se coulent certes dans des formes codifiées qui les légitiment dans une certaine mesure (du moins c'est ce qu'espèrent les manifestants), et se fondent sur des conceptions admises par l'ensemble corps social; mais le jeu avec ces formes, le détournement ou la réinterprétation, voire la redécouverte et la résurrection de pratiques délaissées, ou 
encore la contestation de l'unanimisme communautaire de façade, bref toute la distance que le groupe ou l'individu peut prendre avec son environnement, tout cela existe de nos jours, et a sans doute existé chez les ancêtres des Japonais.

Assez curieusement, Katsumata Shizuo, tout en se focalisant sur les comportements des acteurs dans leurs aspects les plus concrets, individualise peu ses objets d'études : ses moines, guerriers ou paysans sont censés avant tout incarner des types, ou illustrer des attitudes collectives, d'ordre communautaire ou statutaire. Ce n'est que récemment que l'historiographie japonaise a commencé à se pencher sur des acteurs plus personnalisés, mais l'histoire des mentalités promue par Katsumata ne s'intéresse pas encore à ce genre de problématique, pour demeurer dans le cadre de l'étude des «mentalités populaires». On peut d'ailleurs y voir en partie une conséquence de sa spécialisation dans la généalogie du droit médiéval, et de son usage des sources législatives ou réglementaires.

19 L'anthropologie historique dans le sillage d'Amino, après son heure de gloire dans les années 1980, a finalement peiné à renouveler ses problématiques et à répondre aux critiques adressées à sa méthodologie. Elle n'a donc pas véritablement été développée par des successeurs à l'heure actuelle, pour l'histoire médiévale et encore moins pour l'histoire prémoderne. Et la tentative de Katsumata Shizuo d'une lecture globale des mentalités populaires peut sembler aussi isolée, car peu de spécialistes de la société de l'époque d'Edo l'ont suivi sur ce terrain. L'influence des travaux de Katsumata se retrouverait peut-être chez Yasumaru Yoshio, qui continua à s'intéresser aux mouvements et mentalités populaires, à la charnière de la fin du régime shogounal et de l'ère Meiji.

20 Mais l'importance d'Ikki est plutôt dans le coup de grâce que cet ouvrage a donné aux interprétations trop rigides d'une école marxiste d'après-guerre à bout de souffle. Quelques mois avant la publication de l'Ikki de Katsumata, paraissait pourtant aux presses de l'Université de Tōkyō une série d'ouvrages en cinq volumes intitulée elle aussi Ikki, et qui se voulait une somme sur les insurrections populaires; mais en fait le sujet s'épuisait déjà, et la thématique des affrontements paroxystiques entre classes sociales allait progressivement être délaissée au cours des années 1980, au profit d'études plus attentives aux singularités des groupes sociaux et au fonctionnement quotidien des environnements dans lesquels ils évoluaient. Dans ce contexte, l'ouvrage de Katsumata ouvrait l'horizon de l'historiographie du Japon prémoderne sur des nouveaux objets d'études développés par des médiévistes, et délaissés jusqu'alors par les tenants du matérialisme historique. L'influence de Katsumata Shizuo sur les historiens de la période d'Edo ne fut pas immédiate, étant donné que ce milieu restait un bastion du marxisme, plus ou moins orthodoxe il est vrai. Mais il influença sur la durée les esprits des chercheurs et des étudiants, en les préparant à la réception des approches de la Nouvelle Histoire reprises et développées par des historiens comme Ninomiya Hiroyuki.

21 Saluons donc la parution en français de cet ouvrage important pour la compréhension des modes de fonctionnement des institutions communautaires japonaises, et qui se lit de surcroit sans difficulté. La clarté des exposés de Katsumata est parfaitement rendue par l'excellente traduction de Pierre-François Souyri, qui l'a complétée d'un imposant et éclairant appareil de notes, absent au départ, mais indispensable pour un francophone peu familier de l'histoire japonaise. On regrettera juste que quelques traductions de fonctions ou de titres médiévaux (prévôt, etc.), ne soient pas toujours 
accompagnées du terme japonais original, ce qui pourrait intéresser des lecteurs un peu au fait de ces questions. Mais on remerciera Pierre Souyri d'avoir fait précéder sa traduction d'une ample introduction resituant parfaitement le travail de Katsumata dans l'historiographie japonaise contemporaine: même si l'on peut ne pas partager tous les jugements de Pierre Souyri sur certains historiens japonais, cette introduction donne une idée précise de la richesse et de la profondeur des débats historiques au Japon à l'époque contemporaine. L'énorme travail nécessaire pour mener à bien l'édition en français de ce texte de Katsumata suffit à démontrer combien la traduction d'un ouvrage historique de cette importance est en soi une œuvre d'historien, exigeant autant d'effort et de réflexion que l'analyse de sources primaires. Espérons que cette traduction d'Ikki sera suivie de nombreuses autres, qui pourront fournir des éléments pour enrichir un véritable dialogue entre histoire française et japonaise, et faciliter leur insertion non pas seulement dans une world history, mais bien dans une historiographie globalisée.

\title{
AUTEUR
}

\author{
GUILLAUME CARRÉ
}

EHESS-CNRS, UMR 8173 